\title{
Endoscopic Retrieval Devices
}

\author{
Brenna C. Bounds, MD
}

Endoscopy is useful in the clearance of food bolus impactions, retrieval of foreign bodies, and removal of bile duct stones and resected mucosal lesions, which is accomplished by different endoscopic accessories that are passed through the endoscope. This chapter describes these endoscopic retrieval devices, their operation, and clinical applications. Tech Gastrointest Endosc 8:16-21 @ 2006 Elsevier Inc. All rights reserved.

$\mathrm{O}$ vertubes, hoods, and through-the-scope devices such as nets, grasping forceps, snares, pronged grasping devices and baskets are used for endoscopic retrieval of resected tissue specimens, food bolus impactions and foreign bodies.

\section{Overtubes}

\section{Device Description}

Four varieties of disposable overtubes (Guardus ${ }^{\mathrm{TM}}$ Overtube; US Endoscopy, Mentor, $\mathrm{OH}$ ) are currently available, for foreign body extractions and critical cases requiring multiple endoscope insertions, in two lengths and diameters, that fit either a diagnostic or a therapeutic endoscope: (a) esophageal length, standard (OD 8.6-10 mm); (b) esophageal length, therapeutic endoscope (OD 10.00-11.7 mm); (c) gastric length, standard endoscope (OD 8.6-10.0 mm); and (d) gastric length, therapeutic endoscope (OD 10.00-11.7 mm). The Guardus ${ }^{\mathrm{TM}}$ Overtube is a latex-free disposable two-tube system. The tapered tip of inner tube confirms to the size of the endoscope being used, eliminates the gap between the outer diameter of the endoscope and the inner diameter of the overtube during intubation or passage, thus providing a smooth overtube to endoscope transition. The outer tube is reinforced with a metal coil (reduces the risk of tube collapse during use), is transparent (allows visualization of mucosa), and has markings (indicate depth of insertion) and an insuffulation cap (minimizes leakage and allows insuffulation). The distal tip of the overtube expands to accommodate foreign objects being retrieved (Fig. 1).

\section{Device Operation}

After selecting the device with appropriate dimensions to serve the desired therapeutic function, remove the inner tube from the overtube, lubricate both tubes generously with a water soluble lubricant, reassemble the device by inserting the fully lubricated inner tube into the fully lubricated over-

Division of Gastroenterology, Department of Medicine, Massachusetts General Hospital, Harvard Medical School, Boston, MA.

Address reprint requests to Brenna C. Bounds, MD, Blake 453D, 55 Fruit Street, Boston, MA 02114.E-mail: bbounds@partners.org tube, backload an endoscope, and pull the device up to the biopsy port. Insert a bite block to one side of the moth to allow insertion of the endoscope into the esophagus. Once the endoscope is inserted deeply into the esophagus, holding the endoscope shaft straight, slide the overtube assembly down the endoscope shaft into the esophagus using a technique similar to the one routinely used for passing a Savory dilator. Slight amount of rotating motion during overtube insertion aids in a smooth passage through the upper esophageal sphincter. Once the overtube is inserted to a desired length, remove the inner tube and the endoscope simultaneously. After attaching the insuffulation cap to the outer tube, the endoscope is reintroduced through the insuffulation cap. It is critical to avoid pushing the overtube to avoid mucosal tears. Any time the overtube needs to be pushed in, it is advisable to reinsert an endoscope along with the inner tube to prevent trapping of mucosa between the endoscope and overtube. The overtube is useful in removing sharp and long objects. Care must be taken in the insertion of the overtube in patients with a short neck and a fixed neck to avoid respiratory compromise and trauma, respectively.

\section{Latex Protector Hood}

\section{Device Description}

Latex protector hood (Endovation, Reading, PA), an inverted bell-shaped latex device, acts like a hood at the end of the endoscope and protects gastric cardia, esophagus, and posterior pharynx during removal of sharp objects.

\section{Device Operation}

The narrow part of the hood has to be stretched around the tip of the scope and secured to it with a suture. The tip of the endoscope should extend about 2 to $3 \mathrm{~mm}$ into the bell portion of the device to guarantee a clear endoscopic view. After pulling back the bell portion of hood to expose the tip of the scope, the scope-hood assembly is passed into the esophagus as usual. Once a gastric foreign body is located and grasped by one of the devices (snare, basket, or forceps), it is drawn back in contact with the tip of the endoscope. As the instrument is withdrawn through the lower esophageal 

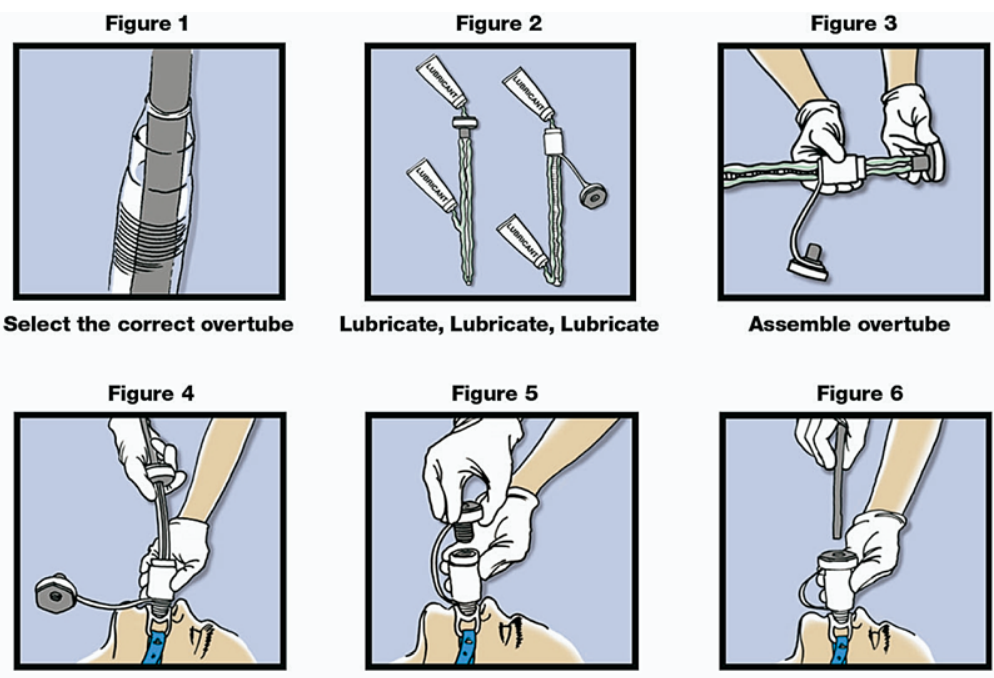

Remove the inner tube an

Attach the insufflation cap

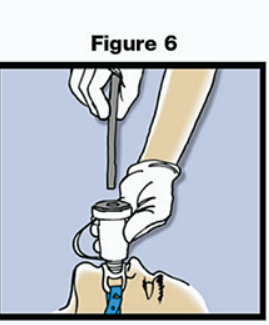

scope simultaneously

Reintroduce the endoscope

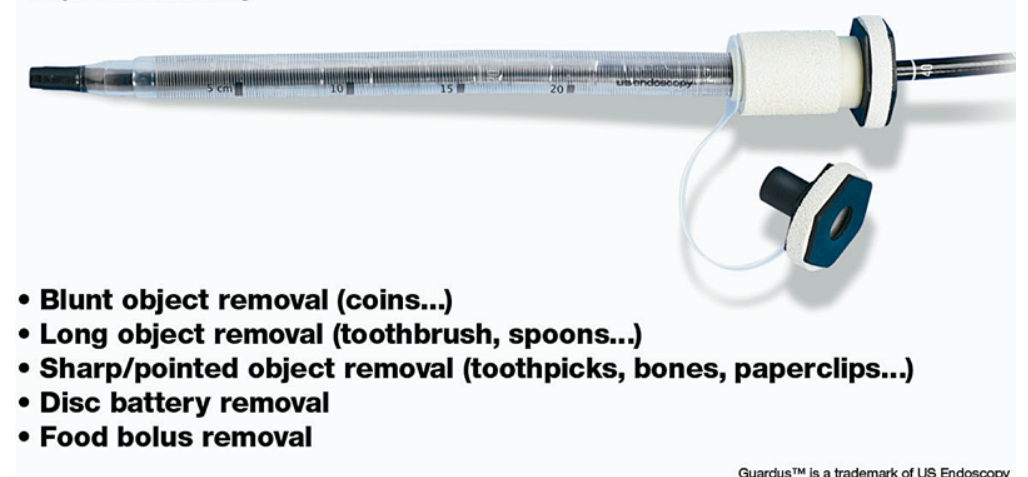

Guardus' $\mathrm{T}$ is a trademark of US Endoscopy
Refer to current Instructions for Use for complete product usage instructions
Courtesy of US Endoscopy

Figure 1 Gaurdus overtube operation and uses.

sphincter, the muscle tension pushes on the hood and flips it back to its original bell-shape hood, which protects the esophagus and pharynx during the retrieval of a sharp object. If the foreign body is primarily located in the esophagus, reinversion of the bell during retrieval is not possible. Therefore, when possible, the foreign body should be pushed into the stomach before removal. If the hood flips back prematurely, it can be reinverted by advancing the endoscope into the first portion of duodenum to reinvert the bell.

\section{Retrieval Nets}

\section{Device Description: Roth Retrieval Nets}

Roth Net (US Endoscopy, Inc.), the first retrieval net designed by Bennett Roth, MD, consists of a snare loop frame to which a small weave net is affixed. Recently, a variety of nets became commercially available: (1) Standard Polyp/Foreign body Roth Net (net size $3 \times 6 \mathrm{~cm}$ ) is useful to retrieve polyps and foreign bodies; (2) Food Bolus Roth Net (net size $4 \times 5.5$ $\mathrm{cm}$ ) is useful to remove food impactions; (3) Pediatric Roth Net (net size $2 \times 4.5 \mathrm{~cm}$ ) is a smaller version of the standard Roth Net; and (4) Maxi Foreign Body Roth Net (net size $4.5 \times$ $7 \mathrm{~cm}$ ) is ideal for large, multiple, and piecemeal polyp removal and large foreign body removal. Different lengths are available for use during EGD, colonoscopy, and enteroscopy (Fig. 2).

\section{Device Operation: Roth Retrieval Nets}

Roth Net has a handle which resembles that of a standard polypectomy snare, and the operation is similar to that of a polypectomy snare. The net is opened and closed in the same way that a polypectomy snare is opened and closed. The excess net forms a pocket during closure of the device which completely encompasses the ensnared object. The pocket permits successful retention of small polyp fragments or foreign bodies as the net is re-opened to permit retrieval of additional material. The object to be retrieved can be easily visualized through the net as the net is positioned over the object and closed.

\section{Device Description: Nakao Snare System}

The Nakao I Snare incorporates both a retrieval net and cutting snare into a single-use double lumen device designed to permit both resection and retrieval of a polyp without exchanging tools. ${ }^{1}$ The Nakao III Cautery Retrieval Snare incorporates both the retrieval net and the snare into a single lumen device. The two loops are constructed in a coplanar configuration, allowing the cautery snare to cut the polyp while the retrieval net secures the specimen simultaneously. There is also a Nakao retrieval net which does not have the cutting snare. 


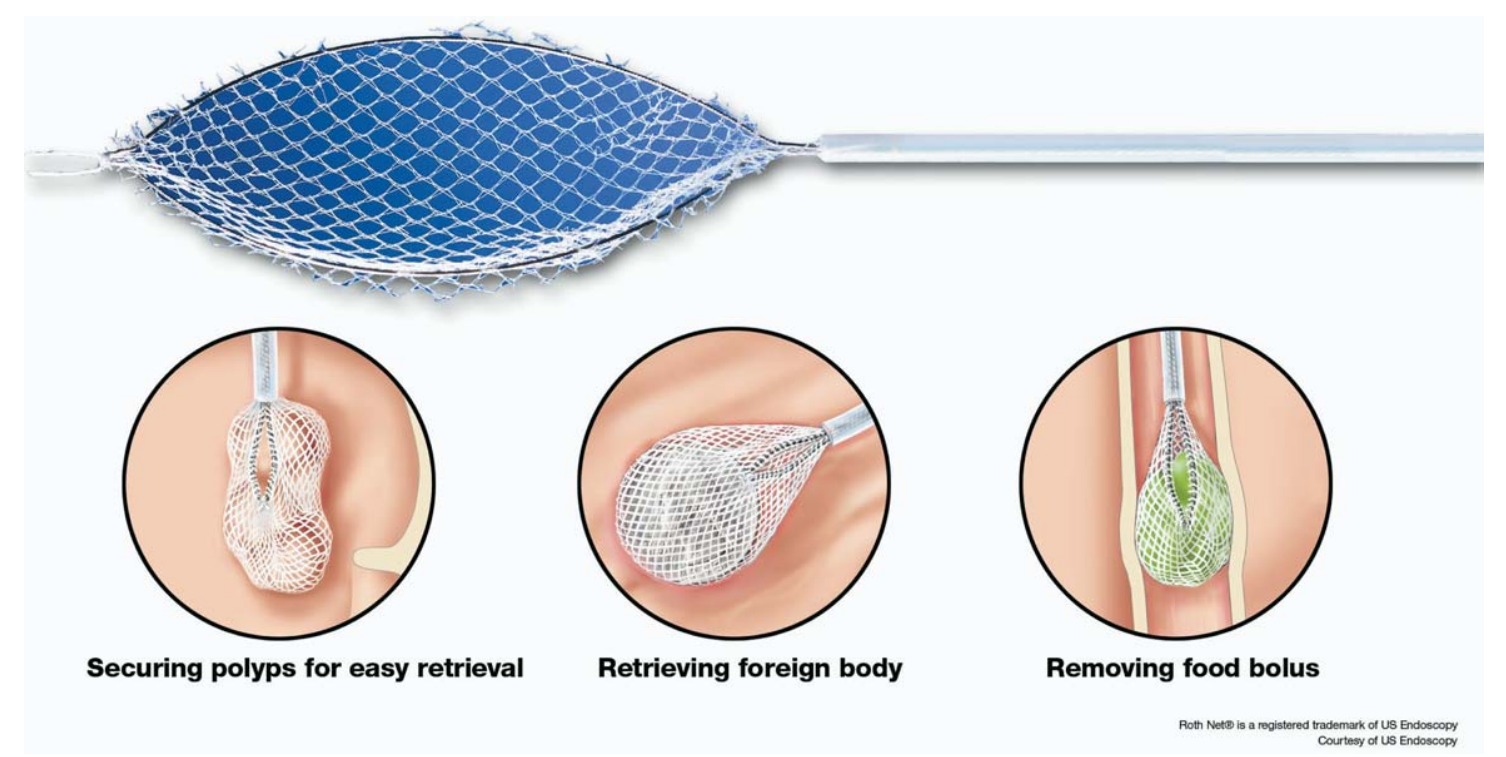

Figure 2 Roth Net uses. (Color version of figure is available online.)

\section{Device Operation: Nakao Snare System}

The Nakao I Snare double lumen system has a handle with a sidearm which opens and closes the retrieval net while the main handle accommodates connection to the cautery unit and controls the opening and closing of the cutting snare. The Nakao III Cautery Retrieval Snare incorporates both the retrieval net and the snare into a signal lumen. The handle of this device resembles a traditional polypectomy snare with optional connection to the cautery unit. Both the snare and the retrieval net can be opened together with one movement of the handle and closed to serve as a one-step approach to polyp resection and retrieval. The Nakao retrieval net operates with a snare technique but does not have the cutting snare. These devices will be distributed by ConMed Endoscopic Technologies beginning in May 2006.

\section{Forceps}

\section{Device Description and Operation Grasping Forceps}

Grasping forceps are available in rat-toothed, alligator, rattoothed alligator, hollow alligator, W-shaped, pelican type, shark tooth, rubber-tipped, and rotatable jaw versions. ${ }^{2}$ Rattoothed forceps have opposing teeth at the tips of the forceps. Alligator jaw forceps have small teeth the length of each of the jaws of the forceps, and rat-toothed alligator jaw forceps are a combination of these two styles. There is also a rotatable version of the rat-toothed alligator jaw forceps. The W-shaped version has longer jaws for gripping larger objects. Pelican type forceps have opposing cups with or without a needle. Shark tooth forceps are similar to alligator forceps with the teeth angled back toward the handle. Rubber-tipped forceps are coated with a nonlatex rubber for gripping small, slippery objects. All of the forceps come in lengths suitable for both upper endoscopes and colonoscopes. A few are available for use through enteroscopes (Fig. 3).

\section{Pronged Grasping Forceps}

Pronged grasping forceps are composed of three to five wires with angled ends. Unlike the standard forceps, the pronged forceps are fully retractable and are contained within a plastic sheath for introduction through the endoscope working channel. They are available both as single use and reusable devices. The handle is designed like that of a polypectomy snare. Care must be taken not to close this type of forceps too aggressively due the flexibility of the wire design. This flexibility makes these ideally suited for polyp retrieval.

\section{Snares}

\section{Device Description and Operation}

Device description and operation of available snares are covered under Snares, Knives, and Scissors (pages 22-27). Standard polypectomy snares should be readily available for endoscopic retrieval of specimens and foreign bodies.

\section{Baskets}

\section{Device Description}

In 1961, Enrico Dormia, a urologist from Italy, developed a basket with four wires for use in retrieving ureteral stones. ${ }^{3}$ Modified for gastrointestinal endoscopic use for retrieval of biliary stones, the baskets have also been used successfully for extraction of foreign bodies, polyps, and food bolus impactions (Fig. 2). Since the initial modification of the urologic basket, many different variations have been developed. ${ }^{4}$ Features of basket such as the shape (Dormia Basket or helical basket), stiffness of wires (stiff wires or pliable wires), opening width of the basket $(22,32$, or $35 \mathrm{~mm}$ ), and presence or absence of additional functions (wire guidance, injection capability, lithotripsy) need to be looked at in the selection of a basket for a particular case.

\section{Device Operation}

The handle of the basket is designed like that of the polypectomy snare, and the same technique is used to open and close the basket. Escape of dye from the bile duct after endoscopic sphincterotomy makes it difficult to locate the stones in the bile duct. This could be overcome by the use of a basket with 


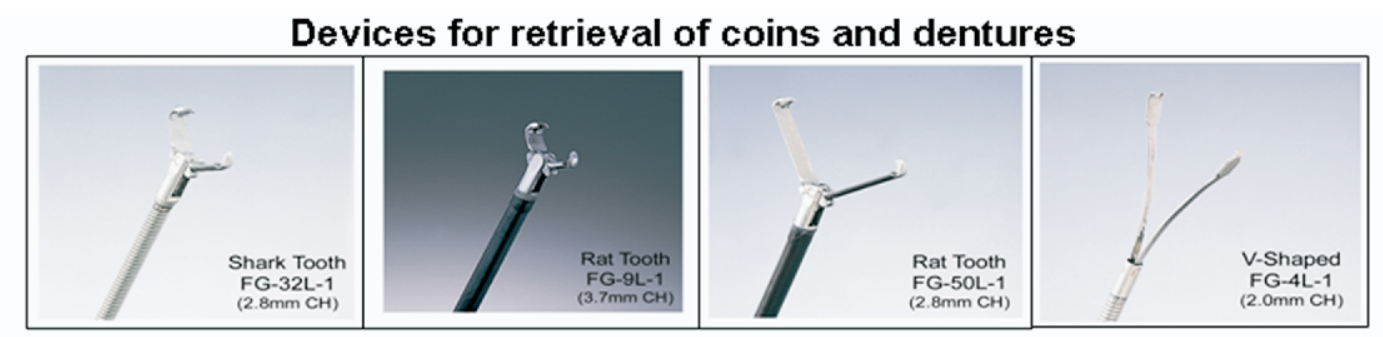

Retrieval of nails, needles, blades, pins, \& other sharp objects

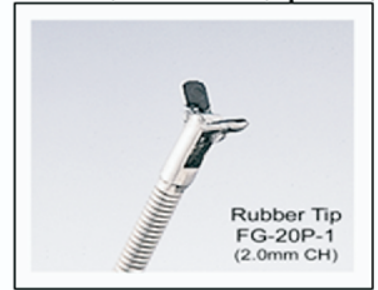

Retrieval of complicated sharp foreign bodies, stents, etc
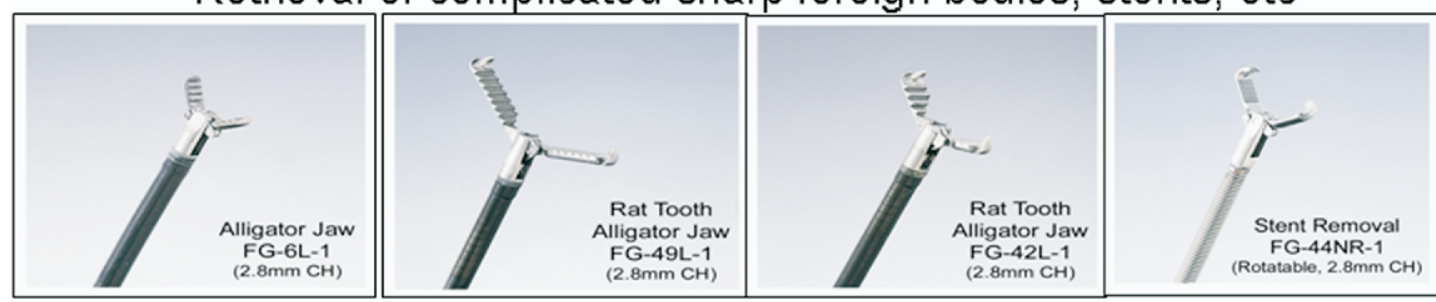

Courtesy of Olympus

Figure 3 Retrieval devices.

an injection port which allows contrast injection to check the location of stones before opening of the basket and capturing of the stones. The stiff basket catheter can easily damage the fresh sphincterotomy site if care is not taken to cannulate the bile duct. A wire-guided basket may facilitate easy cannulation of the sphincterotomy site. In addition, the wire-guided basket facilitates maneuvering the basket around a large biliary stone or foreign body in the esophageal lumen and also into a specific hepatic duct for proximally located stones. In the case of retrieval of large square stones where the risk of basket entrapment is high, soft wire baskets that are compatible with a rescue mechanical lithotripter should be used so that the basket wires can be broken with subsequent removal of the entrapped basket. The handle of lithotripter compatible baskets is removed to expose the wires for use with the rescue lithotripter. Hard wire baskets and spiral baskets are not lithotripter compatible. Use of a hard wire basket for stone extraction should be limited to stones that are no larger than the distal bile duct or sphincterotomy to prevent basket/ stone impaction in the biliary tree to avoid the risk of basket entrapment.

Operation of the basket must be a coordinated effort by the endoscopist and the assistant. As the basket is slowly opened, the endoscopist gently pulls the sheath back to maintain position in the lumen. Once fully opened, the basket is moved to and fro alongside the object to be retrieved until the object is successfully trapped within the wires. Baskets are particularly suited to soft, pigment stones in the biliary tree, which tend to compress into the wall of the bile duct during balloon retrieval and thereby preventing complete clearance of the duct. ${ }^{5}$

\section{Clinical Applications for Endoscopic Retrieval Devices}

\section{Foreign Body Extraction}

The annual incidence of foreign body ingestion in the United States is about 120 per 1 million population, of which approximately 1500 people die as a result of their ingestion. ${ }^{6}$ The majority of foreign body ingestions occur in children between the ages of 6 months and 3 years. ${ }^{7}$ Others at risk include edentulous adults or those with dentures or dental bridge work, psychiatric patients, patients with impaired cognition due to substance abuse, and incarcerated individuals seeking secondary gain.

In most cases, foreign body ingestion or food bolus impaction is a medical urgency that requires endoscopic intervention, and the management depends on the type of foreign body, ie, food-related (meat bolus impaction) and nonfoodrelated, which can be further classified based on the characteristics of the foreign body [blunt or sharp object; corrosive (batteries), or toxic (cocaine)]. ${ }^{7}$ Treatment algorithm is selected based on the nature of ingestion. ${ }^{8}$

It is critical to have the necessary endoscopic accessories for successful removal of any ingested foreign body. In fact, a practice dry run retrieval of an identical or similar foreign body ex vivo to select the most appropriate instrument for grasping should be undertaken for safe, effective, and successful retrieval. ${ }^{7,9}$ Every endoscopy unit should be equipped with the following devices for successful management of ingested foreign bodies: retrieval net, rat tooth and alligator forceps, pronged grasping forceps, Dormia basket, polypectomy snare, foreign body hood, and overtube. ${ }^{10}$ 


\section{Meat Bolus Impaction}

Meat bolus impaction requires emergent endoscopy. If endoscopy is perfomed early, before softening of the meat, extraction of the entire meat bolus can be achieved with a polypectomy snare. If the meat has been present in the esophagus for a longer period of time and has begun to soften, extraction becomes challenging, requiring multiple intubations; using a pronged grasping forceps or Roth Food Bolus retrieval net may prove more effective. An overtube may be considered to minimize hypopharyngeal trauma and protect the airway as pieces of meat are extracted. A pronged grasping forceps or Roth Net may prove useful in this setting. Care must be taken to protect the airway in all cases. If endotracheal intubation is not electively undertaken, placing the patient in a trendelenburg position to prevent the object from dislodging in the trachea is advisable. .,9-11 $^{-1}$

\section{Blunt Foreign Bodies}

Extraction of blunt foreign bodies may be performed with alligator or rat toothed forceps, a retrieval net, a polypectomy snare, or a Dormia basket. Small, smooth objects such as button batteries, marbles, or small coins are best removed using a retrieval net or Dormia basket, which provides secure extraction and protection against inadvertent loss. ${ }^{7,10}$ Larger coins may be successfully retrieved with a W-shaped grasping forceps or polypectomy snare. More complex objects, such as small toys, are best captured and retrieved with a Dormia basket.

\section{Sharp Foreign Bodies}

The most common sharp or pointed foreign bodies are toothpicks, nails, needles, bones, safety pins, straight pins, dental prostheses, and razor blades. ${ }^{7}$ When removing these types of objects, Chevalier Jackson's axiom should be adhered to: "Advancing objects puncture, trailing objects do not." 12 Although sharp or pointed objects lodged in the esophagus represent a medical emergency, once they have passed into the stomach, the majority will traverse the remaining gastrointestinal tract without incident. ${ }^{13}$ Although the overall incidence of gastrointestinal perforation due to foreign body ingestion is less than $1 \%$, sharp and pointed objects result in perforation rates of up to $35 \%$, most commonly in the area of the ileocecal valve. ${ }^{7,14,15}$ For this reason, a sharp or pointed object in the area of the stomach or proximal duodenum should be removed endoscopically if feasible. Administration of $0.5 \mathrm{mg}$ of glucagon intravenously may help to slow motility and aid in retrieval. ${ }^{7}$ Extraction through an overtube or into a foreign body hood should be considered when removing sharp or pointed objects. ${ }^{7,9,10}$ Pins, nails, and tooth picks can be grasped on one end using a rubber tipped forceps. The rubber tip prevents the object from slipping and offers greater security during retrieval. Use of baskets, snares, and retrieval nets should be avoided in most cases, as they offer less control of the sharp object and risk mucosal injury during extraction.

\section{Long Foreign Bodies}

Objects greater than $5 \mathrm{~cm}$ in length present a special challenge, particularly if they are sharp or pointed. One successful technique describes the use of two snares through a dou- ble channel therapeutic gastroscope to grasp and orient a dinner fork before extraction. ${ }^{16}$

\section{Polypectomy and Endoscopic Mucosal Resection}

A more recent clinical application for grasping forceps is the "lift and cut" technique for endoscopic mucosal resection. ${ }^{17,18}$ The mucosal lesion to be removed is "lifted" with a submucosal injection of a solution (saline or saline with an additive such as epinephrine or 50\% dextrose, hypertonic saline, or sodium hyaluronate). A double-channel endoscope allows simultaneous use of both a grasping forceps and cautery snare. The cautery snare is opened first and then the grasping forceps is used to pull the lesion into the open loop. The snare is then closed and the lesion is resected using electric cutting current. The lesion can then be removed with the grasping forceps as the endoscope is withdrawn. The rat-toothed forceps with longer jaws are ideally suited for this technique.

For retrieval of resected gastric, duodenal, and colonic polyps, equipment choices include a pronged grasping forceps, a Dormia basket, polypectomy snare, or retrieval net. ${ }^{4,19-22}$ As previously described, nets are ideally suited to retrieve resected EMR and polyp specimens. ${ }^{23}$ Multiple fragments may be retrieved simultaneously with a net allowing for complete histologic review. ${ }^{23}$ Additionally, use of retrieval net may avoid specimen fragmentation that may preclude margin assessment during histologic evaluation.

\section{References}

1. Nakao NL: Combined cautery and retrieval snares for gastrointestinal polypectomy. Gastrointest Endosc 44:602-605, 1996

2. Nelson DB, Bosco JJ, Curtis WD, et al: ASGE technology status evaluation report. Endoscopic retrieval devices. Gastrointest Endosc 50:932934, 1999

3. Dormia E: Dormia basket: standard technique, observations, and general concepts. Urology 20:437, 1982

4. Waye JD, Lewis BS, Atchison MA, et al: The lost polyp: a guide to retrieval during colonoscopy. Int J Colorectal Dis 3:229-231, 1988

5. Endoscopic therapy of biliary tract and pancreatic diseases. Guidelines for clinical application. Gastrointest Endosc 37:117-119, 1991

6. Devanesan J, Pisani A, Sharma P, et al: Metallic foreign bodies in the stomach. Arch Surg 112:664-665, 1977

7. Webb WA: Management of foreign bodies of the upper gastrointestinal tract: update. Gastrointest Endosc 41:39-51, 1995

8. Benjamin S: Esophageal foreign bodies and perforation, in HaubbrichWSSF (ed): Bockus Gastroenterology. Philadelphia, PA, W.B. Saunders, 1993

9. Guideline for the management of ingested foreign bodies. American Society for Gastrointestinal Endoscopy. Gastrointest Endosc 42:622625,1995

10. Ginsberg GG: Management of ingested foreign objects and food bolus impactions. Gastrointest Endosc 41:33-38, 1995

11. Neustater B, Barkin JS: Extraction of an esophageal food impaction with a Roth retrieval net. Gastrointest Endosc 43:66-67, 1996

12. Jackson CL: Foreign bodies in the esophagus. Am J Surg 93:308-312, 1957

13. Davidoff E, Towne JB: Ingested foreign bodies. N Y State J Med 75 : 1003-1007, 1975

14. Vizcarrondo FJ, Brady PG, Nord HJ: Foreign bodies of the upper gastrointestinal tract. Gastrointest Endosc 29:208-210, 1983

15. Johnson WE: On ingestion of razor blades. J Am Med Assoc 208:2163, 1969

16. Yong PT, Teh $\mathrm{CH}$, Look $\mathrm{M}$, et al: Removal of a dinner fork from the stomach by double-snare endoscopic extraction. Hong Kong Med J 6:319-321, 2000 
17. Akahoshi K, Kojima H, Fujimaru T, et al: Grasping forceps assisted endoscopic resection of large pedunculated GI polypoid lesions. Gastrointest Endosc 50:95-98, 1999

18. Larghi A, Waxman I: Endoscopic mucosal resection: treatment of neoplasia. Gastrointest Endosc Clin North Am 15:431-454, 2005

19. Spencer RJ, Coates HL, Anderson MJ Jr: Colonoscopic polypectomies. Mayo Clin Proc 49:40-43, 1974
20. Webb WA, McDaniel L, Jones L: Experience with 1000 colonoscopic polypectomies. Ann Surg 201:626-632, 1985

21. Wolff WI, Shinya H: Modern endoscopy of the alimentary tract. Curr Probl Surg 1:62, 1974

22. Wolff WI, Shinya H: Colonofiberscopic management of colonic polyps. Dis Colon Rectum 16:87-93, 1973

23. Miller K, Waye JD: Polyp retrieval after colonoscopic polypectomy: use of the Roth Retrieval Net. Gastrointest Endosc 54:505-507, 2001 